\title{
Molecular phylogeny of the Taeniapterini (Diptera: Micropezidae) using nuclear and mitochondrial DNA, with a reclassification of the genus Taeniaptera Macquart
}

\author{
Morgan D. Jackson ${ }^{a, *}$, Stephen A. Marshall ${ }^{a}$, Jeffrey H. Skevington ${ }^{b, c}$ \\ ${ }^{a}$ School of Environmental Sciences, University of Guelph, Guelph, ON, Canada N1G 2W1 \\ ${ }^{b}$ Agriculture and Agri-Food Canada, Canadian National Collection of Insects, Arachnids and \\ Nematodes, Ottawa, ON, Canada K1A 0C6 \\ 'Department of Biology, Carleton University, Ottawa, ON, Canada K1S 5B6 \\ ${ }^{*}$ Corresponding author, e-mail: morgandjackson@gmail.com \\ Published online 31 March 2015, published in print 26 November 2015
}

\begin{abstract}
DNA molecular data are used to generate a phylogeny for the micropezid subfamily Taeniapterinae. Thirtytwo taeniapterine species were sampled, including 10 of the $20 \mathrm{New}$ World genera recognized by Steyskal, as well as one genus formerly treated as a synonym of Poecilotylus Hennig (Hemichaeta Steyskal). Five species from the Micropezinae were included as outgroups. A total DNA dataset of $4705 \mathrm{bp}$, including mitochondrial genes (12S and cytochrome $c$ oxidase I (COI)) and nuclear coding genes (wingless and CAD), was analysed using maximum parsimony and Bayesian inference. The genus Taeniaptera Macquart was found to be non-monophyletic with respect to the remainder of the Taeniapterini analysed here. Taeniaptera is restricted to the Taeniaptera trivittata Macquart species group, Mitromyia Cresson is resurrected to contain the Taeniaptera grata (Wulp) species group, and Paragrallomyia Hendel is resurrected to contain most species previously considered Taeniaptera. Poecilotylus is recognized as a paraphyletic group awaiting further research.
\end{abstract}

Keywords

Taeniapterinae; New World; Taxonomy; Taeniaptera; DNA; Maximum Parsimony; Bayesian Inference

\section{Introduction}

Most genera of Micropezidae are currently diagnosed using a few superficial characters, generally with a high level of variability and homoplasy. The tribe Taeniapterini, for example, is broken into genera based on little more than the shape of the palpus and the type of arista, with most species with a bare arista included in Taeniaptera Macquart (1835) on the basis of an "axe-shaped" palpus. These weak generic concepts need to be tested and either supported and clarified, or replaced by an alternative system of consistently diagnosable monophyletic taxa. We here use DNA data to reconsider the definition of the large genus Taeniaptera and related Taeniapterini. 


\section{Definition and diagnosis of Taeniapterinae and Taeniapterini}

Taeniapterinae is the most diverse subfamily of Micropezidae, and has a global distribution (minus Antarctica), with the greatest diversity occurring in the New World tropics. The subfamily is considered monophyletic based on a suite of apomorphies (ocelli displaced forward; sternopleural (katepisternal) bristles in a dense vertical fan of bristle-like hairs; subscutellum vestigial, not convex; epandrium bilaterally compressed (male); and surstylus absent (male)) (McAlpine 1998). Taeniapterinae is usually divided into two tribes based on the length of cell cup and the length of vein $\mathrm{CuA}_{2}$ relative to vein $A_{1}+C u A_{2}$, with the short-celled genera (all Old World taxa and the following New World genera: Mesoconius Enderlein, 1922; Zelatractodes Enderlein, 1922; Globopeza Marshall 2005; Metasphen Frey, 1927; Scipopus Enderlein, 1922; Pseudeurybata Hennig,1934; Calosphen Hennig, 1934; Grallipeza Rondani, 1850; Planipeza Marshall, 2008; Cliobata Enderlein, 1923; Rainieria Rondani, 1843; Chaetotylus Hendel, 1932; Hoplocheiloma Cresson, 1926) being placed in the Rainieriini of Cresson (1938) (=Grallipezini of Aczél 1951 and subsequent authors), and the exclusively New World long-celled genera (Taeniaptera; Hemichaeta Steyskal, 1968; Parasphen Enderlein, 1922; Ptilosphen Enderlein, 1922; Tenthes Cresson, 1930; Grallomyia Rondani, 1850; Poecilotylus Hennig, 1934; Plocoscelus Enderlein, 1922; Cardiacephala Macquart, 1843) placed in the Taeniapterini (Cresson 1938). We here consider the classification of the tribe Taeniapterini, with a focus on the large genus Taeniaptera and its close relatives. The New World Micropezidae have been studied by some of the leading dipterists of the 20th Century (Cresson 1938, Hennig 1934, 1935a,b, 1936, Aczél 1951, Steyskal 1967, 1968), yet several generic concepts are considered confluent and in need of redefinition; Cliobata and Rainieria; Calosphen and Grallipeza; Pseudeurybata and Scipopus; and, as will be discussed in this study, Taeniaptera and Poecilotylus (Marshall 2010). Taeniaptera (sensu Steyskal 1968) is currently the most diverse of these genera and one of the most problematic taxonomically. Originally erected by Macquart (1835) and redefined by Hennig (1934), this genus included 40 species in Steyskal's (1968) Catalog of Diptera South of the United States. A further four species have since been added (Albuquerque 1980, 1981; Steyskal 1986) without modification of Hennig's definition of the genus. Marshall (2010) followed Hennig (1934) in differentiating Poecilotylus and Taeniaptera by the shape of the maxillary palpus and the frontal vitta, but added wing characters to the diagnoses and expressed doubt that these diagnoses reflected real monophyletic groups. The apparent dearth of consistent morphological characters to define Taeniapterinae genera, possibly in part due to convergence on similar Hymenoptera models, suggests that the classification of this group could be improved with the use of additional, non-morphological character sets. Molecular data have proven useful for understanding generic relationships in other acalyptrate families (Conopidae, Gibson et al. 2010; Sphaeroceridae, Kits et al. 2013; Diopsidae, Baker et al. 2001); we therefore set out to find nuclear and mitochondrial DNA characters of possible use in testing and defining generic boundaries within the Taeniapterini. 


\section{Materials and methods}

\section{Taxon sampling}

Ten of the 20 New World taeniapterine genera recognized by Steyskal (1967) (11 of the 23 New World taeniapterine genera recognized by Marshall, 2010; Marshall \& Jackson, 2014) including six of nine Taeniapterini genera were sequenced, with a particular focus on species of Taeniaptera and Poecilotylus (Table 1). Cardiacephala, Parasphen, and Tenthes were not included due to a lack of suitably preserved material. Cardiacephala is likely closely allied with Plocoscelus, and Parasphen is very similar to Ptilosphen, but Tenthes shows no obvious affinities to other genera. Both genera of Micropezinae (Micropeza Meigen, 1800; Cryogonus Cresson, 1926) were included as outgroups.

\section{DNA extraction and amplification}

Total genomic DNA was extracted, amplified and sequenced following protocols from Gibson et al. (2010); however amplification reactions were carried out in $50 \mu \mathrm{L}$ reactions, and Gibson et al. halved the volume of each component listed compared to our protocol. Primers and polymerase used for each gene sequenced can be found in Table 2.

\section{DNA sequence editing and alignment}

Sequence chromatograms were edited and contigs built using BioEdit ver. 7.0.9.0 (Hall 1999).

Sequence alignments for $12 \mathrm{~S}$ rRNA were performed in ClustalX2 (Larkin et al. 2007) using the default settings. Preliminary alignments for COI, wingless and CAD were performed in ClustalX2 and alignments were then hand-aligned based on amino acid codons using MacClade ver. 4.06 (Maddison et al. 2003). For wingless, a 128 bp unalignable intron was identified at positions 378-506 and excised prior to analysis. No introns were found in the region of CAD sequenced (Moulton \& Wiegmann 2004).

Final sequences were concatenated using TaxonDNA (Vaidya et al. 2011) and formatted for analysis.

\section{Concatenated molecular dataset parsimony analysis}

PAUP* 4.0 beta (Swofford 2003) was used to perform parsimony analyses. Each gene was partitioned and analysed individually, in addition to a total, concatenated analysis. Sequences were added randomly, and a heuristic search using TBR branchswapping for 1000 repetitions. Strict consensus trees were constructed from the most parsimonious trees returned by the analysis, and bootstrap (1000 resampling repetitions, each using 10 random addition sequence repetitions) and jackknife values (deletion frequency $=0.33,1000$ resampling repetitions, each using 10 random addition sequence 


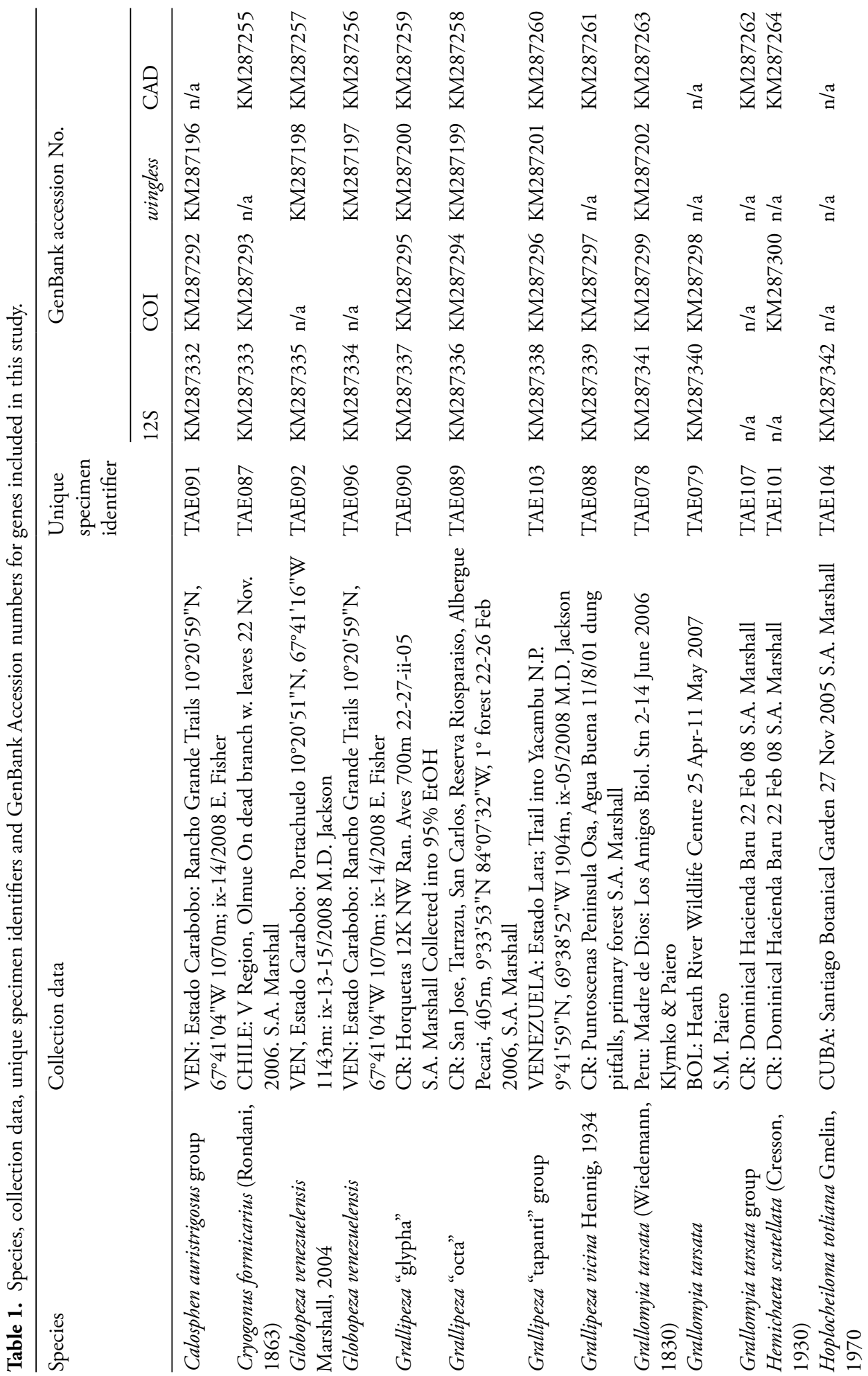




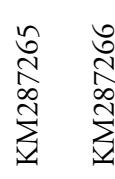

敋

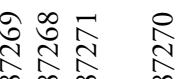

$\infty$ o $\infty$

$\sum_{i}^{\infty} \sum_{i}^{\infty}$

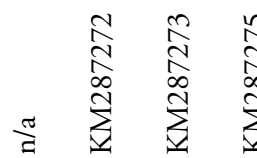

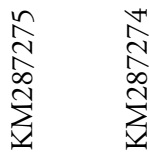

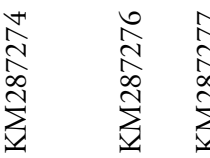

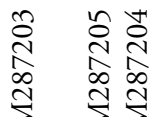

$\stackrel{\sqrt{n}}{\pi} \stackrel{\frac{1}{n}}{\frac{\pi}{n}}$

$\sum \sum \underset{1}{i} \sum_{i}^{\infty}$

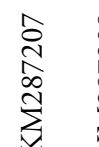

i

$\sum_{i}^{\infty} \sum_{i}^{\infty} \quad \stackrel{N}{\infty} \sum_{i}^{\infty} \sum_{i}^{\infty} \sum_{i}^{\infty}$

ㅇํㅇㅇ

$\sum_{i=1}^{\infty} \sum_{i}^{\infty} \sum^{\infty}$

$\stackrel{\infty}{\infty}$

纱

ָे

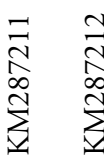

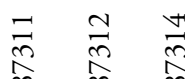

$\stackrel{\infty}{\stackrel{\infty}{*}}$

$\sum_{\substack{\mathbb{N}\\}}^{\infty}$

201

m ॠ

$\sum_{i}^{\infty} \sum_{i}^{\infty} \quad \stackrel{\infty}{\infty} \sum_{i}^{\infty} \sum_{i}^{\infty}$

a) $\infty$

$\sum$

(1)

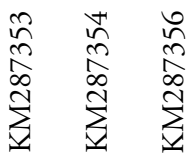

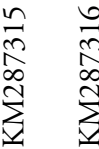

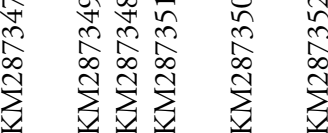

各

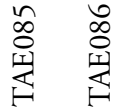

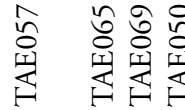

产

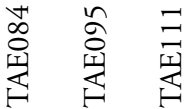

若

Z⿱艹

苟

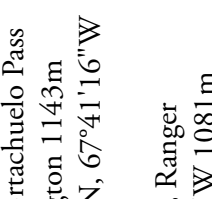

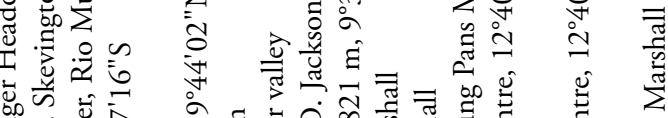

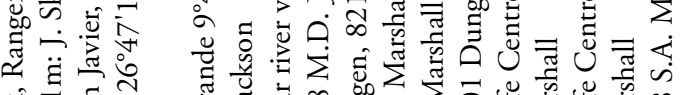

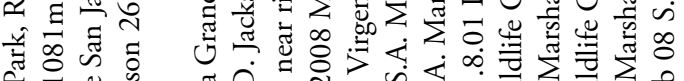

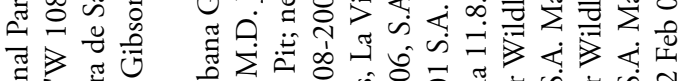

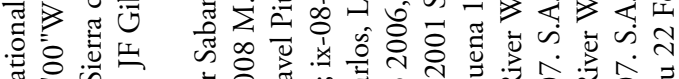

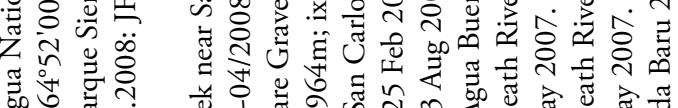

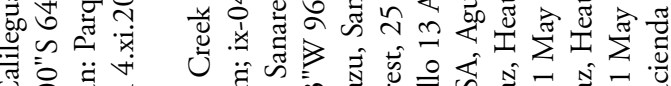
गี

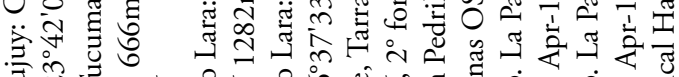

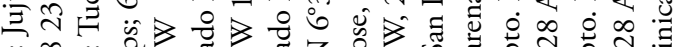

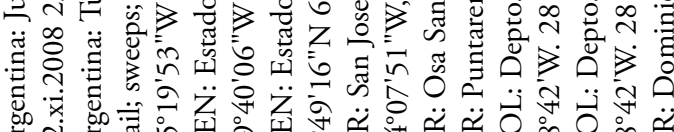

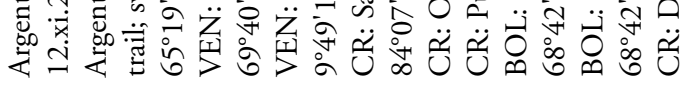

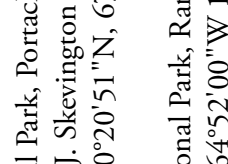

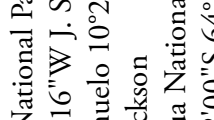

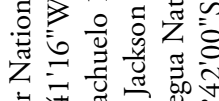

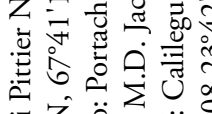

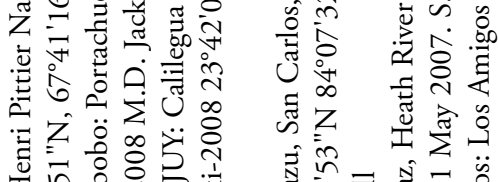

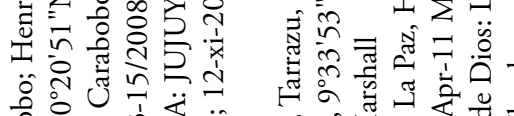

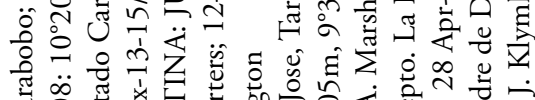

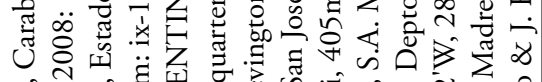

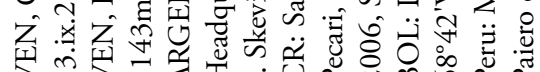

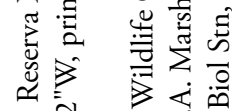

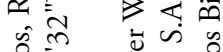

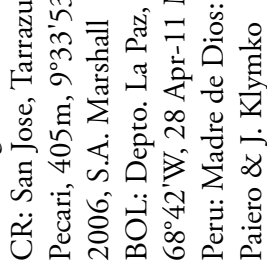

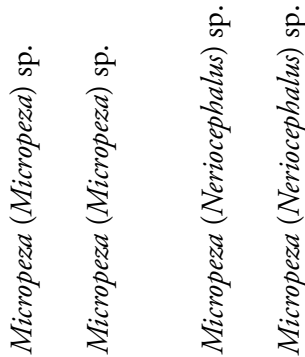

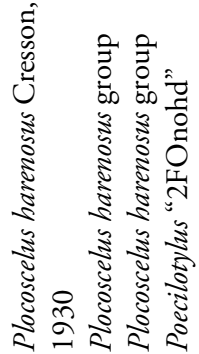

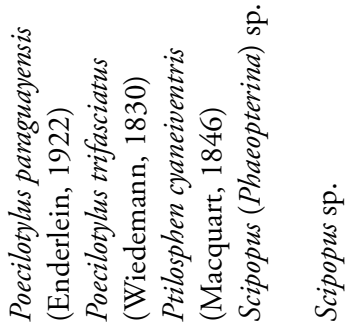

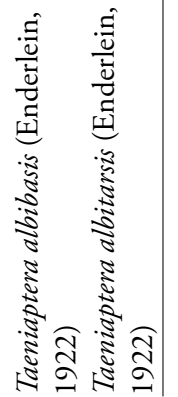




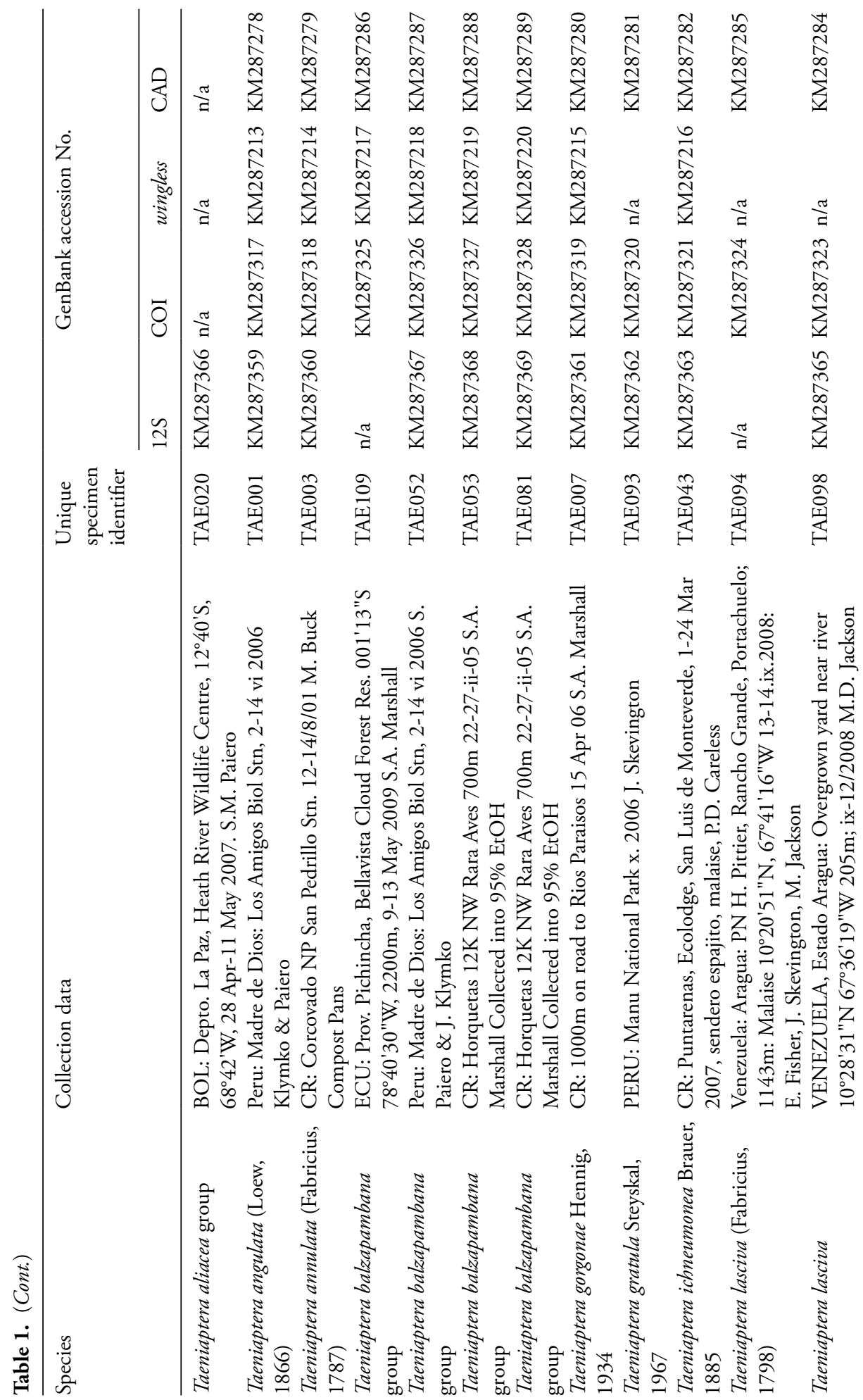




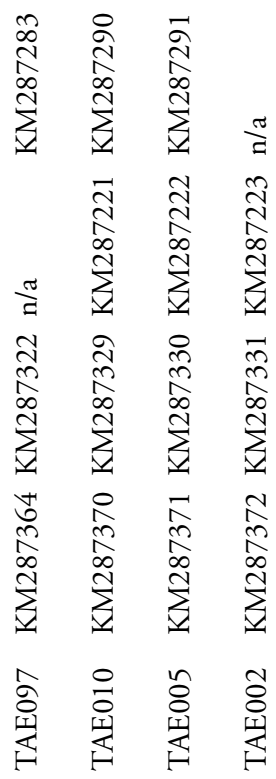

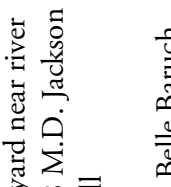

势言

$\cos \sum \sum$

ठ․

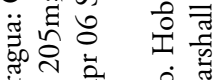

造造 03

을

空

$<ْ$.

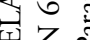

$\mathrm{N}=\tilde{m}$

I1 $\infty$

ก $\ddot{\sim} \quad \ddot{\pi}$

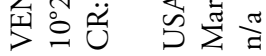

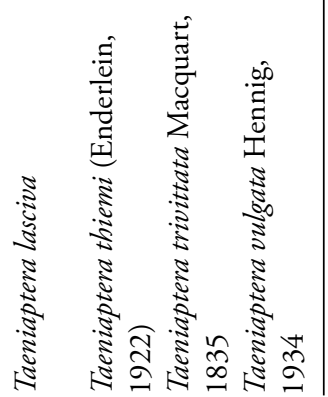




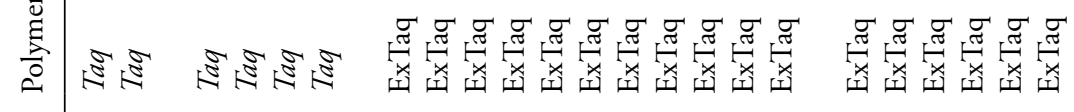

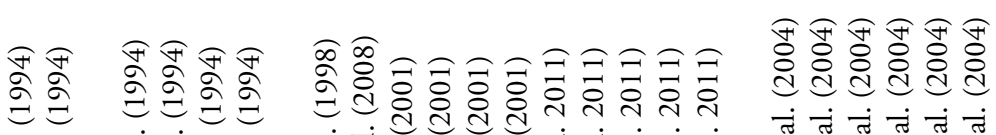

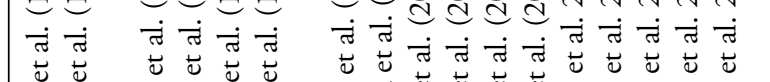

तं तं तं तं तं

च

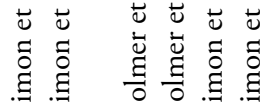

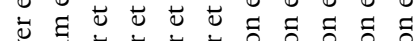

ธี ธี ธี ธี ธี

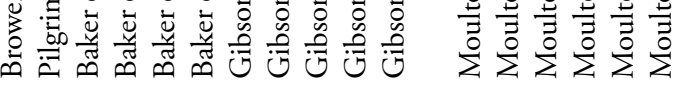

动 的里的

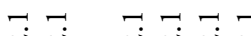

तั

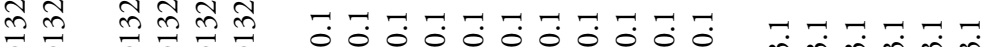

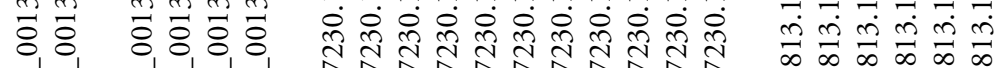

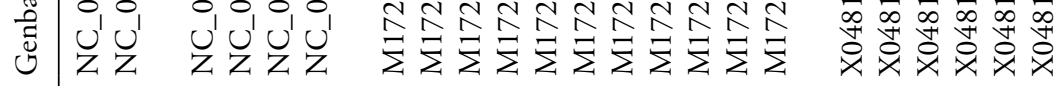

:

.

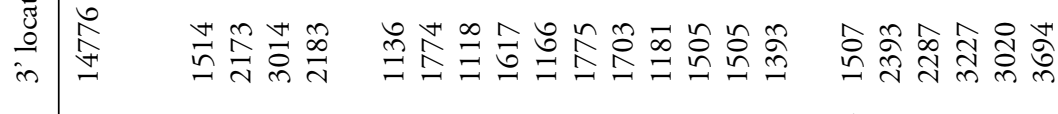

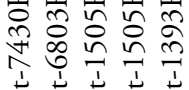

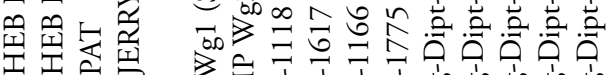

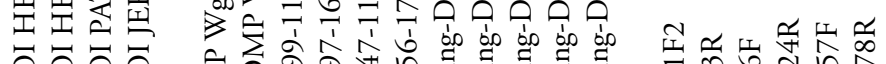

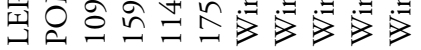

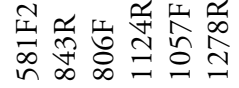


repetitions) were calculated. The full datasets and PAUP* 4.0 scripts used are available from the Dryad Digital Repository (http://dx.doi.org/10.5061/dryad.nn20r). Results of PAUP* 4.0 analyses were confirmed using a New Technologies Search including Sect. Search, Ratchet, Drift, and Tree Fusing with trees generated by randomly adding sequences in TNT: Tree Analysis Using New Technology (Goloboff et al., 2008).

\section{Bayesian inference analysis}

The total concatenated molecular dataset was analysed using Bayesian inference. Data were divided into four partitions (12S, COI, wingless, CAD). MrModeltest ver. 2.3 (Nylander 2004) was used to calculate evolution models of each gene partition in the analysis under Akaike Information Criterion (AIC) (12S, COI, CAD = GTR+I+G; wingless $=\mathrm{K} 80+\mathrm{I}+\mathrm{G})$. Bayesian analysis was performed using $\mathrm{Mr}$. Bayes ver. 3.1.2 (Ronquist \& Huelsenbeck 2003) submitted remotely to the Cyberinfrastructure for Phylogenetic Research Science Gateway V. 3.3 (http://www.phylo.org/index.php/ portal/). The Monte Carlo Markov Chain (MCMC) method was used with the default analysis settings. Four chains (three hot, one cold) were run simultaneously for $1 \times 10^{7}$ generations, with tree sampling occurring every 1000 generations. The first 2500 sample generations were discarded as burn-in. The full dataset and MrBayes command block used are available from the Dryad Digital Repository (http://dx.doi.org/10.5061/ dryad.nn20r).

\section{Results}

\section{$12 S r R N A$}

Sequence data were acquired for 41 taxa. After alignment, a total of 544 bp were analysed, of which 195 (35.8\%) were parsimony informative. Parsimony analysis returned 29 most parsimonious trees (726 steps, $\mathrm{CI}=0.474, \mathrm{RI}=0.646)$, from which a strict consensus tree was constructed (Fig. A1 in the Appendix).

\section{COI}

Sequence data were acquired for 40 taxa. After alignment a total of 1495 bp were analysed, of which 540 (36.1\%) were parsimony informative. Parsimony analysis returned 15 most parsimonious trees (3206 steps, $\mathrm{CI}=0.289$, $\mathrm{RI}=0.483$ ), from which a strict consensus tree was constructed (Fig. A2 in the Appendix).

wingless

Sequence data were acquired for 31 taxa, however the sequence length varied widely between specimens, and a paralog of wingless was found to have been sequenced for Micropezinae. The three micropezine taxa were excluded from the analysis and Globopeza venezuelensis was selected as the outgroup taxon. After alignment, a total of 556 bp were analysed for 28 taxa, of which 135 (24.3\%) were parsimony informative. Parsimony analysis returned 24 most parsimonious trees (418 steps, $\mathrm{CI}=0.583$, 
$\mathrm{RI}=0.668$ ), with which a strict consensus tree was constructed (Fig. A3 in the Appendix).

\section{$C A D$}

Sequence data were acquired for 37 taxa, with a total of 2110 bp analysed, of which $713(33.8 \%)$ were parsimony informative. Parsimony analysis returned four most parsimonious trees (3131 steps, $\mathrm{CI}=0.382, \mathrm{RI}=0.515)$, from which a strict consensus tree was constructed (Fig. A4 in the Appendix).

\section{Concatenated molecular dataset}

DNA sequence data were included from a total of 45 taxa, combining for a total of 4705 bp, 1583 (33.6\%) of which were parsimony informative. Maximum parsimony analysis returned 72 most parsimonious trees ( 7650 steps, $C I=0.382$, $R I=0.515$ ), from which a strict consensus tree was constructed (Fig. 1).

\section{Bayesian inference analysis}

The MCMC chains reached stationarity (split frequencies <0.05) by 436000 generations (final standard deviation of split frequencies 0.0240 , all parameter estimates asymptotic). A total of 7500 generation samples were used to create a majority-rule consensus tree with branch length estimates and posterior probabilities for each node mapped (Fig. 2).

\section{Discussion}

Despite weak backbone congruency between gene trees, Grallomyia was consistently returned as the sister group of a clade, hereafter referred to as Taeniaptera s.s., containing the generic type species Taeniaptera trivittata as well as T. lasciva. A clade containing T. angulata, T. albibasis, T. vulgata, T. annulata, T. gorgonae and T. thiemi, all of which were considered by Hennig (1934) and Steyskal (1968) to be Taeniaptera, was returned separately and is hereafter referred to as the Taeniaptera annulata clade. When the total molecular dataset was analysed, Hemichaeta was returned as the sister group to Grallomyia+Taeniaptera s.s., further complicating "Taeniaptera".

The placement of Hemichaeta and Grallomyia in these analyses renders Taeniaptera non-monophyletic, leaving two possible solutions: synonymize Hemichaeta and Grallomyia with Taeniaptera, or restrict Taeniaptera to Taeniaptera s.s. and recognize the Taeniaptera annulata clade as a separate genus. A broad definition of Taeniaptera encompassing Hemichaeta and Grallomyia would result in a taxon that is very difficult to identify morphologically due to a suite of conflicting characters (shape of the maxillary palpus - parallel-sided in Hemichaeta, axe-shaped in Taeniaptera and Grallomyia; wing cell $\mathrm{r}_{4+5}$ - open to wing margin in the Taeniaptera annulata clade and Grallomyia, closed prior to wing margin in Hemichaeta and Taeniaptera s.s.; length of the sclerotized portion of the distiphallus apical to the phallic bulb - less than $50 \%$ of the 


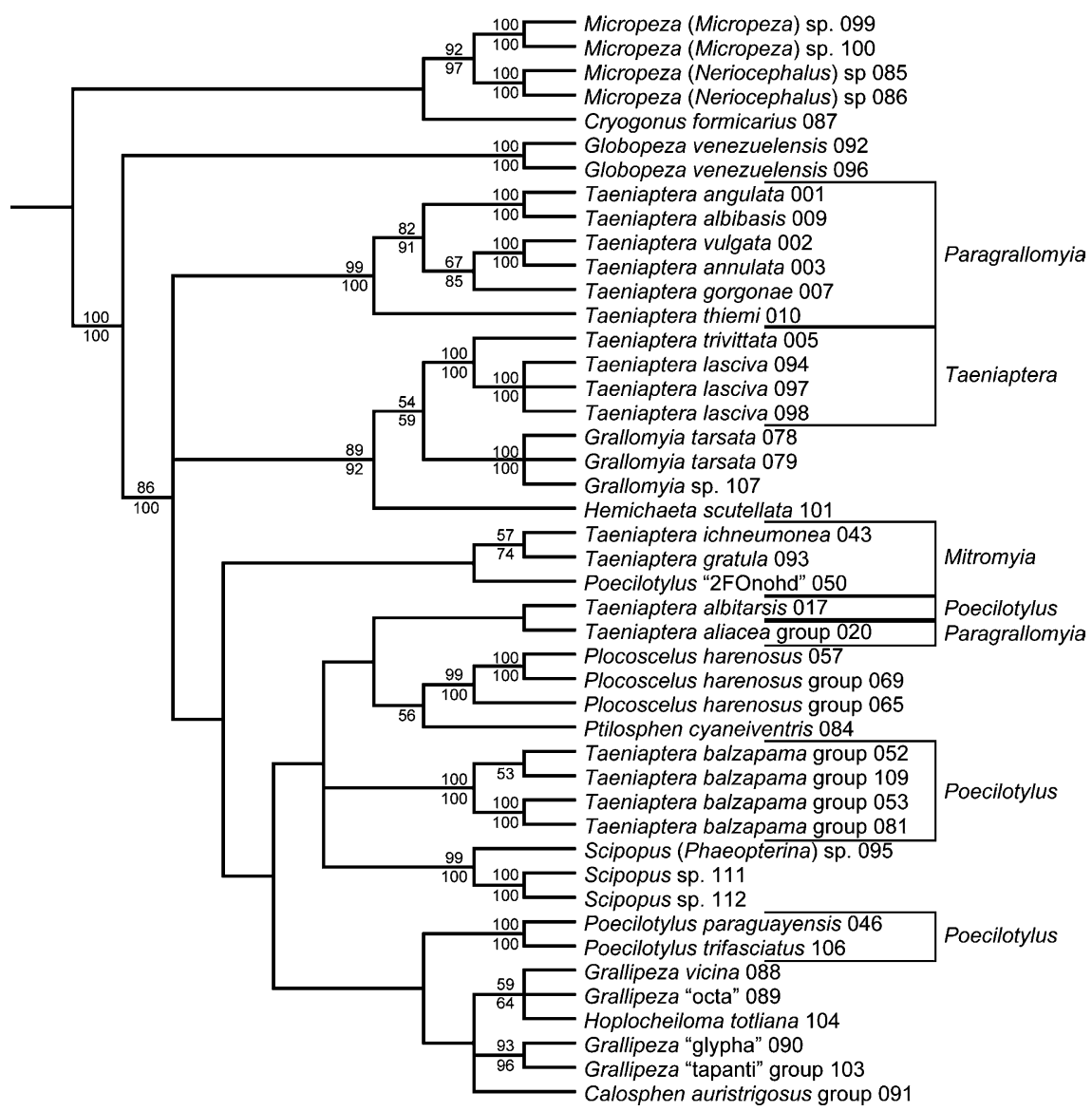

Fig. 1. Strict Consensus Tree for the Taeniapterini from concatenated DNA sequence dataset of four genes (12S, COI, wingless, CAD) (constructed from 72 most parsimonious trees; tree length=7650 steps, $\mathrm{CI}=0.382$, $\mathrm{RI}=0.515$ ). Nomenclature of taxon labels follows current published generic concepts (Steyskal, 1968; Marshall, 2010; Marshall \& Jackson, 2014). Numbers following taxon name indicate unique specimen identifiers for this study. New generic classifications proposed as a result of this study are indicated by labeled brackets. Numbers above nodes represent Bootstrap support, numbers below nodes represent Jackknife support.

length of the basal distiphallus in the Taeniaptera annulata clade and Grallomyia, greater than $50 \%$ of the length of the basal distiphallus in Hemichaeta and Taeniaptera s.s.).

Alternatively, Taeniaptera can be restricted to the Taeniaptera trivittata clade, requiring recognition of the species in the Taeniaptera annulata group as a separate genus. Hendel (1933) already provided a name for that group when he erected Paragrallomyia as a subgenus of Grallomyia, designating Taeniaptera annulata (as Grallomyia) as the type species. The elevation of Paragrallomyia to the generic level is the most acceptable option based on the taxa considered in this paper, since it breaks the former genus into two diagnosable clades, each with an existing name, while leaving the easily diagnosed taxa Hemichaeta and Grallomyia as separate genera. Paragrallomyia can be recognized 


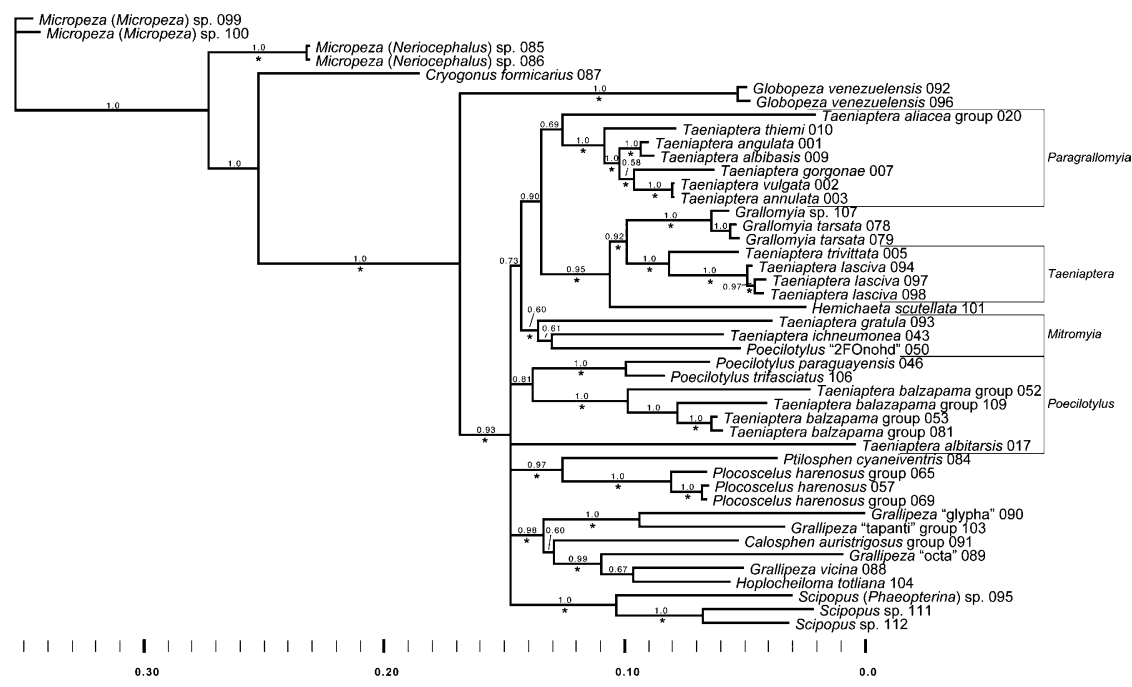

Fig. 2. Majority Rule Consensus cladogram from Bayesian Markov Chain Monte Carlo analysis of the Taeniapterini from concatenated DNA sequence dataset (12S, COI, wingless, CAD), including branch lengths, $\mathrm{RI}=0.483$ ). Nomenclature of taxon labels follows current published generic concepts (Steyskal, 1968; Marshall, 2010; Marshall \& Jackson, 2014). Numbers following taxon name indicate unique specimen identifiers for this study. New generic classifications proposed as a result of this study are indicated by labeled brackets. Numbers at each node represent posterior probabilities. ${ }^{*}$ Nodes recovered in the maximum parsimony analysis (Fig. 1).

morphologically by an axe-shaped maxillary palpus, wing cell $r_{4+5}$ open to the wing margin, and by the presence of at least one dorsocentral bristle.

Hemichaeta scutellata is currently the only species placed in Hemichaeta (Marshall \& Jackson 2014). A relationship between Hemichaeta and Taeniaptera is suggested by some morphological characters including wing venation $\left(\mathrm{R}_{4+5}\right.$ and $\mathrm{M}_{1+2}$ converging well before the wing margin, forming a closed cell $\mathrm{r}_{4+5}$ ) and distiphallus proportions and shape (portion distal to the phallic bulb more than $50 \%$ length of the segment basal to the phallic bulb and strongly hooked towards the posterior). Neither of these character states are present in Grallomyia (wing cell $\mathrm{r}_{4+5}$ open to the wing margin; distiphallus beyond bulb much shorter than basal portion, not strongly hooked towards the posterior). Grallomyia is currently monotypic, although at least one further species awaits description. Marshall (2010) recognized Grallomyia as a distinct genus in his key to Neotropical Micropezidae, and we support that nomenclatural decision here.

Our data show that, as expected, some species previously considered to be in Taeniaptera are misplaced. Among the species included in this analysis, Taeniaptera albitarsus was consistently found to be widely separated from the Taeniapteral Paragrallomyia clade despite a superficial similarity to Paragrallomyia species. We propose a transfer of Taeniaptera albitarsus to Poecilotyus to reflect this relationship, while at the same time recognizing that Poecilotylus is currently a paraphyletic assemblage of unplaced species and is in need of revision. The Taeniaptera aliacea group specimen we 
included was returned outside of Taeniaptera/Paragrallomyia in the maximum parsimony analysis, but as sister to Paragrallomyia using Bayesian inference. Until this species complex can be further studied, we propose retaining it in Paragrallomyia.

Taeniaptera gratula was also recovered outside of the Taeniapteral Paragrallomyia clade, along with Taeniaptera ichneumonea and a similar undescribed species. These species, along with Taeniaptera grata Wulp, 1897, T. wulpi Steyskal, 1967 and T. feei Steyskal 1986 form a distinctive clade that keys out with Poecilotylus in Marshall (2010); however, there is an available name that applies to this clade, Mitromyia Cresson 1930 (type species Mitromyia conifer Cresson 1930 = Calobata grata Wulp 1897). Steyskal (1967, 1986) considered Taeniaptera grata, T. gratula, T. wulpi, and later Taeniaptera feei to form a well-defined group characterized by unusual posterodorsal humps on the head, as well as synapomorphic male genital characters (Steyskal 1986), and stated "Should further investigation of the large and multifarious genus Taeniaptera lead to means of defining and delimiting distinct genera, then Mitromyia may be found to deserve generic rank" (1986: p. 175). We agree, and therefore resurrect Mitromyia to include Taeniaptera grata, T. gratula, T. wulpi, T. feei and T. ichneumonea.

Poecilotylus sensu Marshall (2010) was recovered as paraphyletic, as predicted by Marshall (2010), including two or three distinct species groups depending on analysis method. Poecilotylus is currently a convenient repository for Taeniapterini that do not fit into defined monophyletic taxa such as Taeniaptera ss, Paragrallomyia, Grallomyia, Hemichaeta, Plocoscelus, or Ptilosphen. All nomenclatural changes proposed as a result of these analyses can be found in Table 3.

The Grallipeza species included in these analyses displayed considerable variation in their phylogenetic positions. The four species included in this analysis represent disparate species groups of what is currently considered Grallipeza, a genus currently undergoing a full revision to complement Marshall's Caribbean clade revision (2013). Grallipeza and Calosphen together represent a taxonomic mire similar to the Taeniapteral Poecilotylus problem examined in this study, as neither genus is currently adequately defined or consistently diagnosable.

Our data do not support the tribal classifications proposed by Cresson (1938) and Aczél (1951), with the Taeniapterini rendering the Rainieriini/Grallipezini paraphyletic, however they remain a convenient shorthand for the division of the subfamily Taeniapterinae into two phenetic groups based on distinctive wing venation characters. We suggest the retention of these tribes for now, with the full expectation that further phylogenetic analysis of the subfamily will provide additional evidence that one or both tribes are an artificial group.

\section{Acknowledgements}

The authors would like to thank Scott Kelso and Joel Gibson for assistance with lab work and DNA sequence acquisition, as well as Joel Kits, Gil Gonçalves-Miranda, Andrew Young and Steve Paiero for general help with phylogenetics and discussions 
Table 3. Changes to generic nomenclature of the Taeniapterini based on phylogenetic relationships.

Mitromyia Cresson, 1930 resurrected from synonymy

Mitromyia grata (Wulp, 1897) (type species)

Mitromyia gratula (Steyskal, 1967) comb.n.

Mitromyia ichneuomoneus (Brauer, 1885) comb.n.

Mitromyia munda (Wulp, 1897) comb.n.

Mitromyia wulpi (Steyskal, 1967) comb.n.

Paragrallomyia Hendel, 1933 stat.n., resurrected from synonymy

Paragrallomyia aeripennis (Enderlein, 1922) comb.n.

Paragrallomyia albibasis (Enderlein, 1922) comb.n.

Paragrallomyia aliacea (Albuquerque, 1980) comb.n.

Paragrallomyia angulata (Loew, 1866) comb.n.

Paragrallomyia annulata (Fabricius, 1787) (type species)

Paragrallomyia caerulescens (Macquart, 1846) comb.n.

Paragrallomyia continentalis (Hennig, 1934) comb.n.

Paragrallomyia dilutimacula (Enderlein, 1922) comb.n.

Paragrallomyia diversicolor (Macquart, 1855) comb.n.

Paragrallomyia feei (Steyskal, 1986) comb. n.

Paragrallomyia gorgonae (Hennig, 1934) comb.n.

Paragrallomyia inornata (Hennig, 1934) comb.n.

Paragrallomyia inpai (Albuquerque, 1980) comb.n.

Paragrallomyia latifascia (Wulp, 1897) comb.n.

Paragrallomyia lauta (Cresson, 1930) comb. n.

Paragrallomyia longifurca Hendel, 1933

Paragrallomyia mediofusca (Hennig, 1934) comb.n.

Paragrallomyia nigritarsis (Macquart, 1848) comb.n.

Paragrallomyia parens (Cresson, 1926) comb.n.

Paragrallomyia planitibia (Enderlein, 1922) comb.n.

Paragrallomyia platycnema (Loew, 1866) comb.n.

Paragrallomyia postannulus (Enderlein, 1922) comb.n.

Paragrallomyia rufifacies (Macquart, 1851) comb.n.

Paragrallomyia seiuncta (Czerny, 1931) comb.n.

Paragrallomyia simillima (Hendel, 1922) comb.n.

Paragrallomyia strigata (Enderlein, 1922) comb.n.

Paragrallomyia teresacristinae (Albuquerque, 1981) comb.n.

Paragrallomyia thiemi (Enderlein, 1922) comb.n.

Paragrallomyia tibialis (Macquart, 1843) comb.n.

Paragrallomyia vittipennis (Coquillet, 1902) comb.n.

Paragrallomyia volens (Cresson, 1926) comb.n.

Paragrallomyia vulgata (Hennig, 1934) comb.n.

Poecilotylus Hennig, 1934

Poecilotylus albitarsis (Enderlein, 1922) comb.n.

Poecilotylus balzapambana (Enderlein, 1922) comb.n.

Poecilotylus bistrigatus (Enderlein, 1922)

Poecilotylus buscki (Cresson, 1930)

Poecilotylus egregius (Hennig, 1934)

Poecilotylus leucomelas (Walker, 1852)

Poecilotylus lividisoccatus (Enderlein, 1922)

Poecilotylus luridilabris (Enderlein, 1922) 
Table 3. (Cont.)

Poecilotylus minor (Hennig, 1934)

Poecilotylus obliquus (Fabricius, 1805)

Poecilotylus paraguayensis (Enderlein, 1922)

Poecilotylus pictus Hennig 1937

Poecilotylus stellatus (Wulp, 1897)

Poecilotylus testaceus (Fabricius, 1805)

Poecilotylus tricolorus (Enderlein, 1922)

Poecilotylus trifasciatus (Wiedemann, 1830)

Poecilotylus varius (Wiedemann, 1830) (type species)

Taeniaptera Macquart, 1835

Taeniaptera lasciva (Fabricius, 1798)

Taeniaptera nigriceps Hennig 1934

Taeniaptera trivittata Macquart 1835 (type species)

Taeniaptera vulpes Cresson 1926

regarding evolution of the Diptera. Funding for this project was provided by NSERC Discovery Grants to S.A.M. and J.H.S., a US National Science Foundation Assembling the Tree of Life grant (EF-0334948) to B.M. Wiegmann, funding to J.H.S. from Agriculture and Agri-Food Canada and a North American Dipterists Society travel grant to M.D.J.

\section{References}

Aczél, M.L. (1951) Morfologia Externa Y Division Sistematica de Las Tanypezidiformes: Con synopsis de las especies Argentinas de Tylidae (Micropezidae) Y Neriidae (Dipt.). Acta Zoologica 11: 483-589, pls. $1-4$.

Baker, R.H., Wilkinson, G.S. \& DeSalle, R. (2001) Phylogenetic Utility of Different Types of Molecular Data Used to Infer Evolutionary Relationships among Stalk-Eyed Flies (Diopsidae). Systematic Biology 50: 87-105.

Cresson, E.T. (1930) Notes on and descriptions of some Neotropical Neriidae and Micropezidae. Transactions of the American Entomological Society 56: 307-362.

Cresson, E.T. (1938) The Neriidae and Micropezidae of America North of Mexico. Transactions of the American Entomological Society 64: 293-366, pls. XI-XIII.

de Albuquerque, L.P. (1980) Estudo dos Micropezideos da Bacia Amazônica II - Redescrição de três aspécies e conhecimento de duas espécies novas para a ciênca de Taeniaptera Macquart, 1835 (DipteraMicropezidae). Acta Amazonica 10: 863-881.

de Albuquerque, L.P. (1981) Estudo dos Micropezideos da Bacia Amazônica III - Conhecimento de uma nova espécie e redescrição de três espécies de Taeniaptera Macquart, 1835 (Diptera - Micropezidae). Acta Amazonica 11: 821-838.

Folmer, O., Black, M., Hoeh, R. Lutz, R. \& Vrijenhoek, R. (1994) DNA primers for amplification of mitochondrial cytochrome $\mathrm{c}$ oxidase subunit I from diverse metazoan invertebrates. Molecular Marine Biology and Biotechnology 3: 294-299.

Gibson, J.F., Skevington, J.H. \& Kelso, S. (2010) Placement of the Conopidae (Diptera) within Schizophora based on mtDNA and nrDNA gene regions. Molecular Phylogenetics and Evolution 56: 91-103.

Gibson, J.F., Kelso, S., Jackson, M.D., Kits, J.H., Miranda, G.F.G. \& Skevington, J.H. (2011) Dipteraspecific PCR-amplification primers of use in molecular phylogenetic research. Annals of the Entomological Society of America 104: 976-997. 
Goloboff, P.A., Farris, J.S. \& Nixon, K.C. (2008) TNT, a free program for phylogenetic analysis. Cladistics 24: 774-786.

Hall, T.A. (1999) BioEdit: a user-friendly biological sequence alignment editor and analysis program for Windows 95/98/NT. Nucleic Acids Symposium Series 4: 95-98.

Hendel, F. (1933) Ueber einige Typen Wiedemann's und Schiner's von acalyptraten Musciden aus Südamerika, nebst einigen verwandten Arten (Dipt.). Revista de Entomologia 3: 58-83.

Hennig, W. (1934) Revision der Tyliden (Dipt., Acalypt.). I. Teil: Die Taeniapterinae Amerikas. Stettiner Entomologische Zeitung 95: 65-108, 294-330.

Hennig, W. (1935a) Revision der Tyliden (Dipt., Acalypt.). I. Teil: Die Taeniapterinae Amerikas [concl.]. Stettiner Entomologische Zeitung 96: 27-67.

Hennig, W. (1935b) Revision der Tyliden (Dipt., Acalypt.). II. Teil: Die außeramerikanischen Taeniapterinae, die Trepidariinae und Tylinae. Allgemeines über die Tyliidaen. Konowia 14: 68-92, 192-216, 289-310.

Hennig, W. (1936) Revision der Tyliden (Dipt., Acalypt.) II. Teil: Die außeramerikanischen Taeniapterinae, die Trepidariinae und Tylinae. Allgemeines über die Tyliidaen. Konowia 15: 129-144.

Kits, J.H., Marshall, S.A. \& Skevington, J.H. (2013) Phylogeny of the Archiborborinae (Diptera: Sphaeroceridae) Based on Combined Morphological and Molecular Analysis. PLoS ONE 8: e51190.

Larkin, M.A., Blackshields, G., Brown, N.P., Chenna, R., McGettigan, P.A., McWilliam, H., Valentin, F., Wallace, I.M., Wilm, A., Lopez, R., Thompson, J.D., Gibson, T.J. \& Higgins, D.G. (2007) Clustal W and Clustal X version 2.0. Bioinformatics 23: 2947-2948.

Macquart, P.J.M. (1835) Histoire Naturelle des Insectes - Dipteres. Vol. 2. Librairie Encyclopédique de Roret, Paris, 778 pp.

Maddison, D.R. \& Maddison, W.P. (2003). MacClade. Sinauer Associates, Sunderland, MA.

Marshall, S.A. (2010) 55: Micropezidae (stilt-legged flies). In: Brown, B.V., Borkent, A., Cumming, J.M., Wood, D.M., Woodley, N.E. \& Zumbado, M. (Eds) Manual of Central American Diptera Vol. 2. National Research Council Canada, Ottawa, ON, pp. 805-813.

Marshall, S.A. (2013) Grallipeza Rondani (Diptera: Micropezidae: Taeniapterinae) of the Caribbean and North America. Zootaxa 3682: 45-84.

Marshall, S.A. \& Jackson, M.D. (2014) A redefinition of the genus Hemichaeta, new status (Diptera; Micropezidae; Taeniapterinae). Zootaxa 3815: 291-296.

McAlpine, D.K. (1998) Review of the Australian stilt flies (Diptera: Micropezidae) with a phylogenetic analysis of the family. Invertebrate Taxonomy 12: 55-134.

Moulton, J.K. \& Wiegmann, B.M. (2004) Evolution and phylogenetic utility of CAD (rudimentary) among Mesozoic-aged Eremoneuran Diptera (Insecta). Molecular Phylogenetics and Evolution 31: 363-378.

Nylander, J.A.A. (2004) MrModeltest v2. Evolutionary Biology Centre, Uppsala University, Uppsala. Program distributed by the author.

Ronquist, F. \& Huelsenbeck, J.P. (2003) MRBAYES 3: Bayesian phylogenetic inference under mixed models. Bioinformatics 19: 1572-1574.

Simon, C., Frati, F., Beckenbach, A., Crespi, B., Liu, H. \& Flook, P. (1994) Evolution weighting, and phylogenetic utility of mitochondrial gene sequences and a compilation of conserved polymerase chain reaction primers. Annals of the Entomological Society of America 87: 651-701.

Steyskal, G.C. (1967) Notes on some older types of Neotropical Micropezidae with descriptions of new species (Diptera: Acalyptratae). Papéis Avulsos de Zoologia 20(8): 75-84.

Steyskal, G.C. (1968) Family Micropezidae. In: P.E. Vanzolini and N. Papavero. (Eds) A Catalogue of the Diptera of the Americas South of the United States. Departmento de Zoologia, Secretaria da Agricultura, Sau Paulo, pp. 48.1-48.33.

Steyskal, G.C. (1986) A new species of Taeniaptera from the United States (Diptera: Micropezidae). Proceedings of the Entomological Society of Washington 88: 174-178.

Swofford, D.L. (2003) PAUP*. Phylogenetic Analysis Using Parsimony (*and Other Methods). Version 4. Sinauer Associates, Sunderland, MA.

Vaidya, G., Lohman, D.J., Meier, R. (2011) SequenceMatrix: concatenation software for the fast assembly of multi-gene datasets with character set and codon information. Cladistics 27: 171-180. 


\section{Appendix}

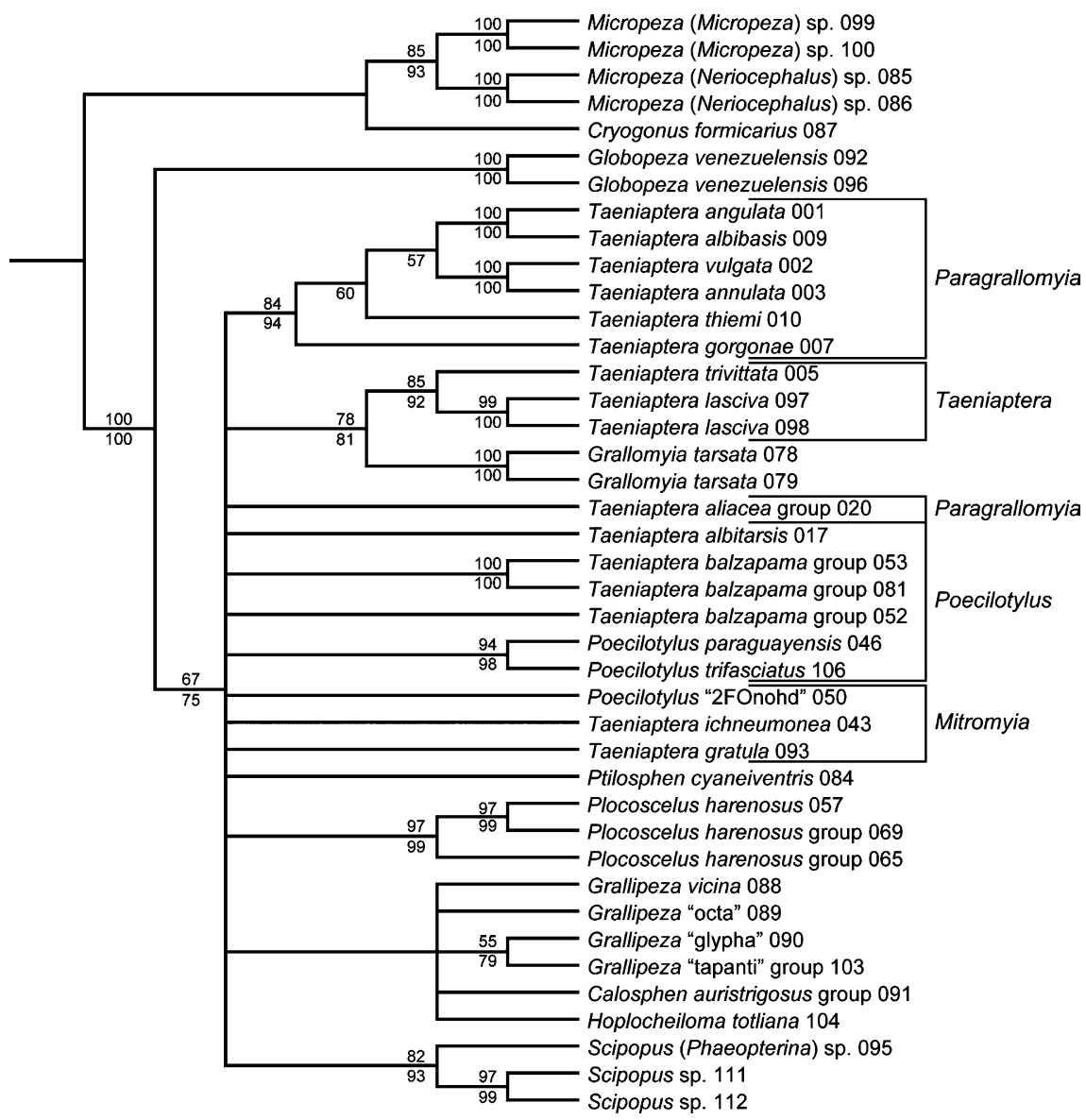

Fig. A1. Strict Consensus Tree for the Taeniapterini from 12S sequence data (constructed from 29 most parsimonious trees; tree length $=726$ steps, $\mathrm{CI}=0.474, \mathrm{RI}=0.646$ ). Nomenclature of taxon labels follows current published generic concepts (Steyskal, 1968; Marshall, 2010; Marshall \& Jackson, 2014). Numbers following taxon name indicate unique specimen identifiers for this study. New generic classifications proposed as a result of this study are indicated by labeled brackets. Numbers above nodes represent Bootstrap support, numbers below nodes represent Jackknife support. 


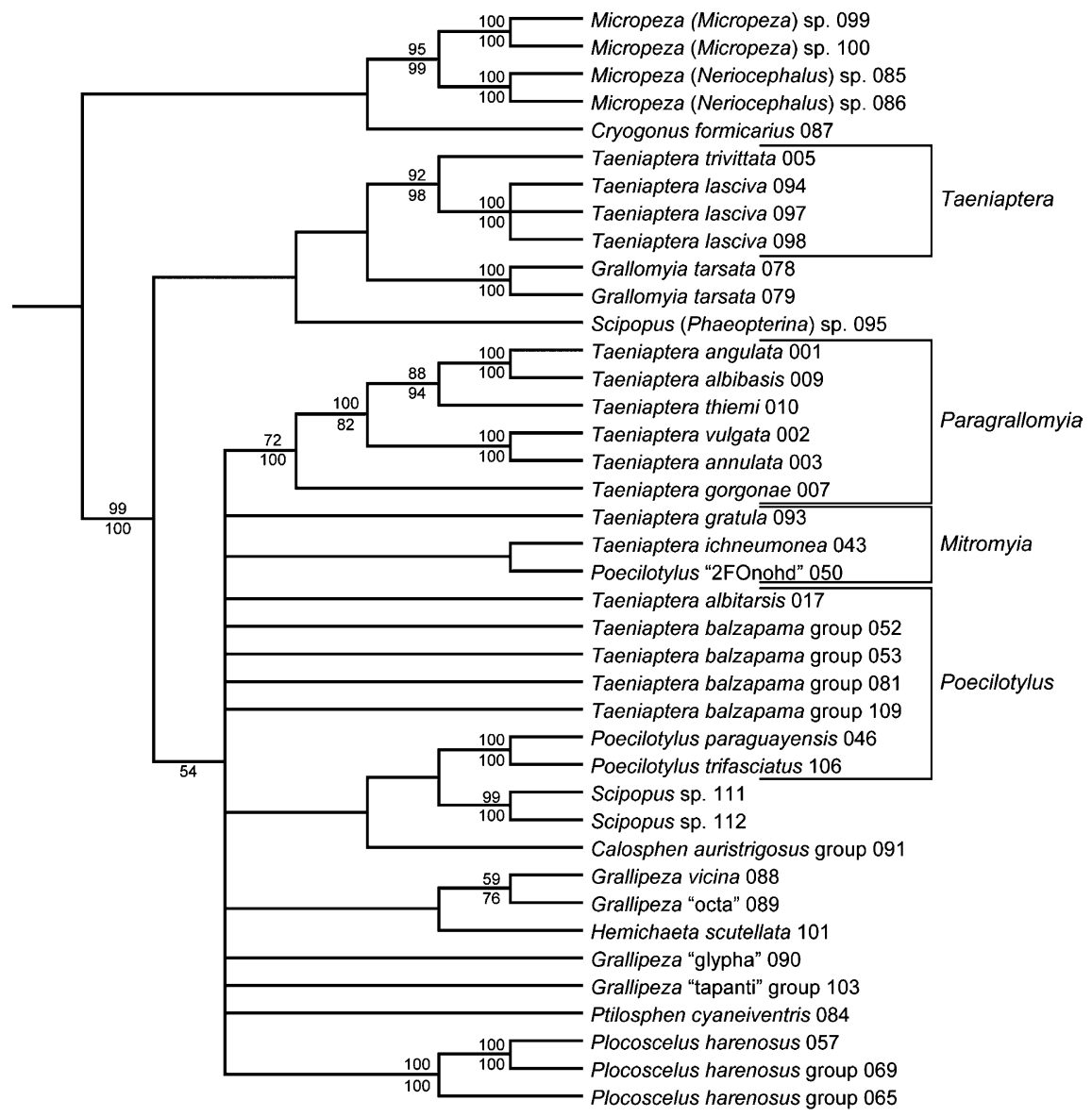

Fig. A2. Strict Consensus Tree for the Taeniapterini from COI sequence data (constructed from 15 most parsimonious trees; tree length=3206 steps, $\mathrm{CI}=0.289$, $\mathrm{RI}=0.483$ ). Nomenclature of taxon labels follows current published generic concepts (Steyskal, 1968; Marshall, 2010; Marshall \& Jackson, 2014). Numbers following taxon name indicate unique specimen identifiers for this study. New generic classifications proposed as a result of this study are indicated by labeled brackets. Numbers above nodes represent Bootstrap support, numbers below nodes represent Jackknife support. 


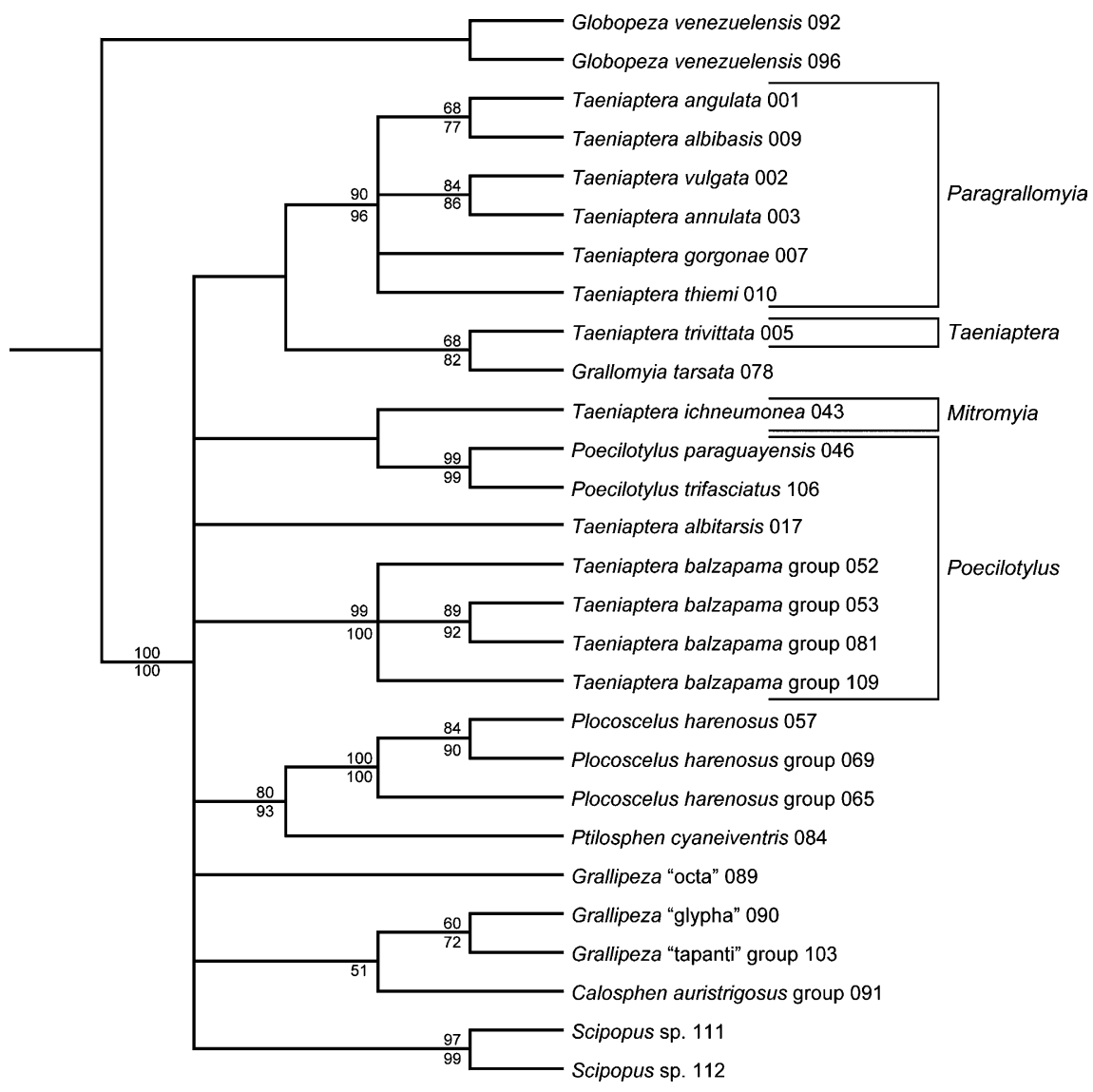

Fig. A3. Strict Consensus Tree for the Taeniapterini from wingless sequence data (constructed from 24 most parsimonious trees; tree length $=418$ steps, $\mathrm{CI}=0.584, \mathrm{RI}=0.668$ ). Nomenclature of taxon labels follows current published generic concepts (Steyskal, 1968; Marshall, 2010; Marshall \& Jackson, 2014). Numbers following taxon name indicate unique specimen identifiers for this study. New generic classifications proposed as a result of this study are indicated by labeled brackets. Numbers above nodes represent Bootstrap support, numbers below nodes represent Jackknife support. 


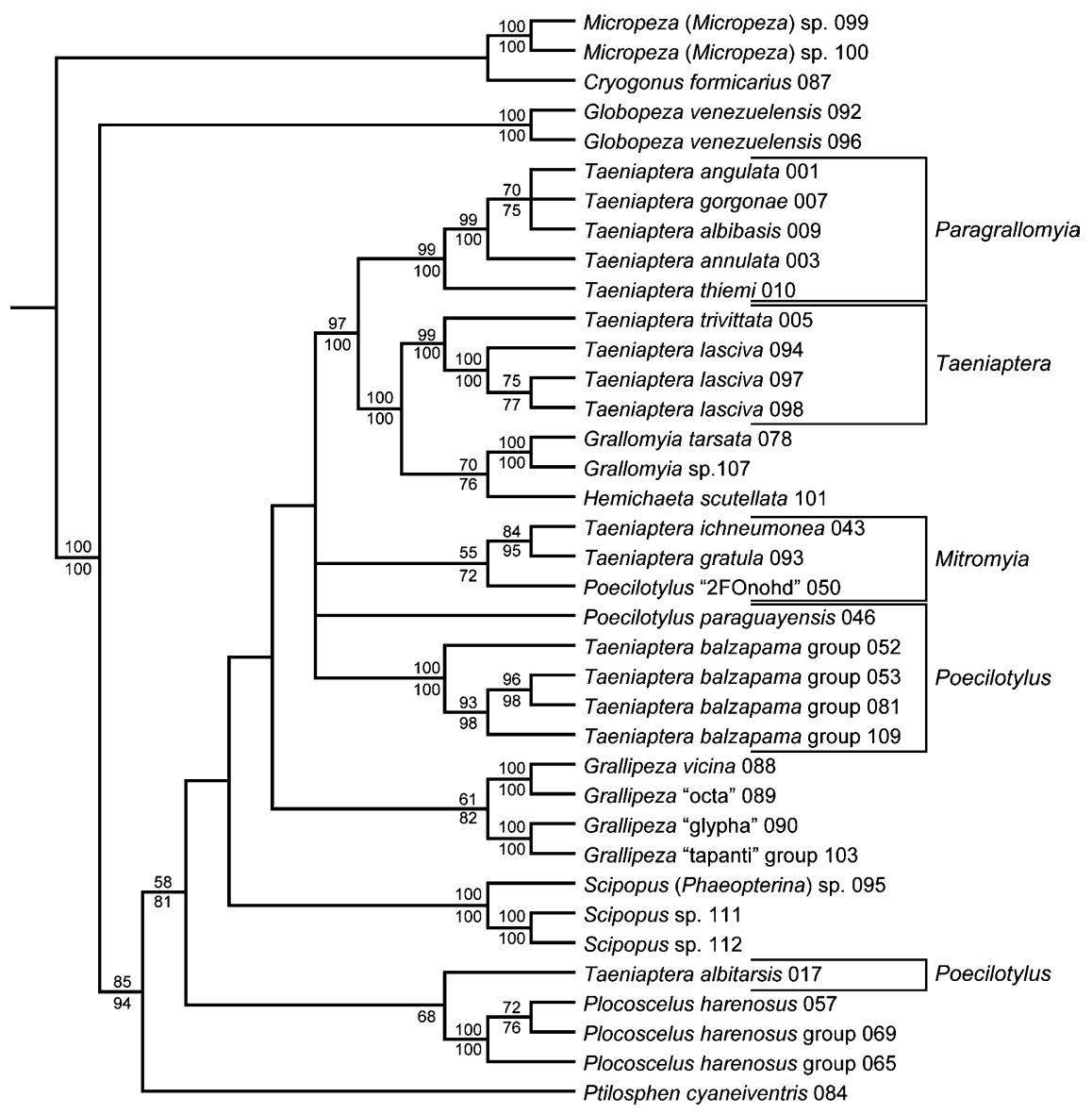

Fig. A4. Strict Consensus Tree for the Taeniapterini from CAD sequence data (constructed from 4 most parsimonious trees; tree length $=3131$ steps, $\mathrm{CI}=0.451, \mathrm{RI}=0.540$ ). Nomenclature of taxon labels follows current published generic concepts (Steyskal, 1968; Marshall, 2010; Marshall \& Jackson, 2014). Numbers following taxon name indicate unique specimen identifiers for this study. New generic classifications proposed as a result of this study are indicated by labeled brackets. Numbers above nodes represent Bootstrap support, numbers below nodes represent Jackknife support. 